Check for updates

Cite this: RSC Adv., 2017, 7, 53018

Received 11th September 2017 Accepted 2nd November 2017

DOI: $10.1039 / c 7 r a 10092 b$

rsc.li/rsc-advances

\section{Carbon nanotube/polyurethane films with high transparency, low sheet resistance and strong adhesion for antistatic application}

\author{
Ying Tian, (DD a Xingcai Zhang, (DD ${ }^{b}$ Hong-Zhang Geng, (D) *ab Hai-Jie Yang, ${ }^{c}$ \\ Chungang Li, ${ }^{d}$ Shi-Xun Da, ${ }^{a}$ Xiushan Lu, ${ }^{d}$ Jie Wang ${ }^{a}$ and Song-Lin Jia (D) ${ }^{a}$
}

\begin{abstract}
Antistatic technology has been applied in all aspects of modern life, including the manufacture of electronic products, aerospace systems, daily necessities and so on. The main purpose of this study is to obtain, using the Mayer rod-coating method, a flexible antistatic film with high transmittance, low sheet resistance and strong adhesion. With the help of the dispersant, single-walled carbon nanotubes (SWCNTs) were dispersed in water to form an homogeneous dispersion. The SWCNT dispersion was coated onto a poly(ethylene terephthalate) (PET) film with use of a rod to produce a uniform film. The antistatic films obtained had special characteristics, such as high transparency, low sheet resistance and excellent resistance to water and heat. Sheet resistance varied between $10^{2}-10^{5} \Omega \mathrm{sq}^{-1}$ by controlling the content of water-based polyurethane (WPU), changing the thickness of the films and the post-treatment. The best film had a sheet resistance of $423 \Omega \mathrm{sq}^{-1}$ with $82.7 \%$ transmittance. In particular, the addition of the WPU binder greatly improved the adhesion between the CNTs and the PET film. Scanning electron microscopy, energy dispersive $\mathrm{X}$-ray spectroscopy and Scotch ${ }^{\mathrm{TM}}$ tape method were conducted to determine the microstructure, cleanliness, and adhesion of the film, respectively.
\end{abstract}

\section{Introduction}

With the development of various productions, antistatic technology has been widely used in productions and life including plastic packaging materials. Improving the antistatic properties of plastic packaging materials will contribute to the development of electronic products, aerospace applications and other fields. Poly(ethylene terephthalate) (PET) films are one of the commonly used packaging films. Because of their high transparency, excellent mechanical properties, ${ }^{1-3}$ gas barrier ${ }^{4}$ and vapor barrier, ${ }^{5}$ the PET films have many packaging applications including food packaging films, electronic products packaging films, display device packaging films, and so on. In recent years, PET packaging films have been widely used in thin film transistors, ${ }^{6}$ solar cells, ${ }^{7}$ and so on. As packaging film, PET films have a lot of inherent advantages, but PET films can also carry electrostatic charge or discharge easily during the process of production and transportation because of the accumulation of

${ }^{a}$ State Key Laboratory of Separation Membranes and Membrane Processes, Tianjin Key Laboratory of Advanced Fibers and Energy Storage, School of Materials Science and Engineering, Tianjin Polytechnic University, Tianjin 300387, PR China. E-mail: genghz@tjpu.edu.cn; Fax: +86 22 83955055; Tel: +86 2283955812

${ }^{b}$ Department of Electrical Engineering and Computer Science, Massachusetts Institute of Technology, Cambridge, Massachusetts 02139, USA. E-mail: genghz@mit.edu

'Tianjin Polyester New Material Technology Engineering Center, Tianjin Wanhua Co., Ltd, Tianjin, 300385, PR China

${ }^{d}$ Tianjin BoYuan New Materials Co. Ltd., Tianjin 300384, PR China static electricity resulting from electrical insulation, ${ }^{8}$ which can cause explosions and difficulties in the sealing of powder products, especially in the packaging of electronic devices. So research on improving the antistatic properties of PET packaging films will have a significant role on our daily lives.

At present, the antistatic materials mainly include carbon black, ${ }^{9}$ carbon nanotubes (CNTs),${ }^{10}$ carbonaceous fibers, ${ }^{11}$ metals and metal oxides, ${ }^{12,13}$ antistatic agents, ${ }^{14-17}$ conductive polymers, ${ }^{18-20}$ and so on. Although the use of metals and metal oxides can give high transparent antistatic films (TAFs), the cost is too high for general use. Conductive film made by adding an antistatic agent easily loses its efficacy, because the antistatic agent easily dissolves in water or loses from the substrate which can lead to contamination and static failure. Although carbon black is widely used as an antistatic agent, it is not the ideal choice because of its poor conductive properties and relatively poor dispersion. Because the conductive polymer itself has some shortcomings, such as poor rigidity, low transparency, and difficulty in forming, it cannot be used as a packaging film directly and ideally should be blended with another polymer matrix. However, properties, such as transparency, flexibility and tensile properties, of the polymer matrix may be affected by adding a large amount of conductive polymer that is used to improve the electrical properties of the films. CNTs, are a onedimensional conductive material with excellent mechanical properties and electrical conductivity, which is widely used in transparent conducting thin films, ${ }^{21-24}$ chemical sensors, ${ }^{25}$ and 
light-emitting diodes, ${ }^{26,27}$ and so on. In antistatic applications, many researchers have utilized CNTs as a conductive material to make antistatic films. Huang et al. made use of multi-walled carbon nanotubes (MWCNTs) and cellulose to make films on glass, but the films did not have good conductivity or flexibility. ${ }^{28}$ Yoon et al. used in situ polymerization to make composites containing MWCNTs and poly(ethylene), and then used them to make films with a surface resistance of $10^{10} \Omega$, which was high enough for them to be used in practical applications. ${ }^{29} \mathrm{Kim}$ et al. utilized MWCNTs and isopropyl alcohol to make films on poly(ethylene naphthalate). Using this method, the resistance decreased, but the transmittance was between $55 \%$ to $60 \%$ at the wavelength of $550 \mathrm{~nm}$, which was too low. ${ }^{30}$ Kitamura et al. made a composite film containing aligned CNTs, but the surface resistance of this film was not uniform in all directions. ${ }^{31}$ For antistatic films, it is better to improve various properties at the same time. A method to fabricate antistatic films was found which could simultaneously have properties with good performances, such as transmittance, low sheet resistance, flexibility, large area, uniformity, and strong adhesion at room temperature. Single-walled carbon nanotubes (SWCNTs) have better conductivity than MWCNTs but have poor adhesion to the PET substrate, which caused short-lived antistatic film and pollution. To solve this problem, a water-based poly(urethane) (WPU) was chosen as a binder to improve the adhesion. Comparing organic solvent-based poly(urethane)s, WPU has many advantages such as non-toxicity, it is environmentally friendly, has high adhesion $^{32}$ and it has been widely used in adhesives, coating materials, ${ }^{33-35}$ and so on.

In this research, SWCNTs were used as the conductive material and WPU as the binder to improve the adhesion between the CNTs and the PET substrate. The low-power bar coating technology, which can easily control the film thickness and has many advantages including its simplicity, it does not require use of raw materials, it keeps the nature of the original films, and was adapted to obtain large area, uniform, transparent and conductive films at room temperature. Finally, the TAFs made of CNTs were further post-treated to reduce the sheet resistance. Scanning electron microscopy (SEM), energy dispersive X-ray spectroscopy (EDX), and the Scotch ${ }^{\mathrm{TM}}$ tape method were conducted to determine the properties of the TAFs. The results obtained showed that this method gave films which gave a good performance in antistatic applications.

\section{Experimental}

\subsection{Preparation of CNT solution}

High purity SWCNTs ( $95 \mathrm{wt} \%$, diameter $<2 \mathrm{~nm}$, length of 5-30 $\mu \mathrm{m}$ ) grown using chemical vapor deposition were purchased from the Chengdu Organic Chemicals Co. Ltd. China. Sodium dodecylbenzenesulfonate (SDBS) and Triton X-100 (TX-100) were purchased from Aladdin and used as received. The WPU mass fraction of $30 \%$ was obtained from Tianjin BoYuan Advanced Materials Co. Ltd, China and used as received. SWCNTs $\left(1 \mathrm{mg} \mathrm{ml}^{-1}\right)$, SDBS ( $\left.1 \mathrm{wt} \%\right)$ and deionized water were placed in to a vial bottle. The suspension was mixed ultrasonically in a bath sonicator for $10 \mathrm{~min}$, and then sonicated in a probe sonicator for $70 \mathrm{~min}$ followed by centrifugation at $8000 \mathrm{rpm}$ for $15 \mathrm{~min}$. TX-100 and WPU were added into the SWCNT-SDBS dispersion and stirred at $65{ }^{\circ} \mathrm{C}$ for $10 \mathrm{~min}$ in a water bath. A stable CNT suspension was obtained for the production of the TAFs. The schematic in Fig. 1 shows the preparation of the CNT suspension, coating method, posttreatment and characterization of TAFs.

\subsection{Coating method}

The PET films were produced by Tianjin Wanhua Co., Ltd, China with an average thickness of $75 \mu \mathrm{m}$. First of all, the PET film was soaked in acetone for $30 \mathrm{~min}$, dried in an oven at $70{ }^{\circ} \mathrm{C}$ and then fixed on a temperature controlled heating plate or a glass plate at room temperature. CNT suspension $(0.2 \mathrm{ml})$ was drawn in a uniform line over the fixed PET film from left to right using a straw, and then the line was spread up and down the PET film using a Mayer rod equipped with a wire with a diameter of $0.2 \mathrm{~mm}$ or $0.3 \mathrm{~mm}$ corresponding to a wet film thickness of $18 \mu \mathrm{m}$ or $27 \mu \mathrm{m}$, respectively. The uniform CNT film was dried at $70{ }^{\circ} \mathrm{C}$ for $1-2 \mathrm{~min}$. The CNT films obtained were described as CNT-TAFs.

\subsection{Post-treatment of CNT-TAFs}

The CNT-TAFs obtained were immersed in deionized water for $10 \mathrm{~min}$ and rinsed for 2-3 times to remove most of the SDBS and TX-100 on the surface of the CNTs and this was followed by drying at $80{ }^{\circ} \mathrm{C}$ until the CNT-TAFs were completely dry. The resulting TAFs were described water washed CNT-TAFs. Then the water washed CNT-TAFs were immersed in $12 \mathrm{M}$ of nitric acid for $30 \mathrm{~min}$ to further remove the residual SDBS and TX-100 on the surface of the CNTs to obtain acid treated CNT-TAFs. Different contents of WPU were added to the CNT dispersion to explore the impact of WPU on the sheet resistance of the CNT-TAFs. The content of WPU was optimized to obtain CNTTAFs with high adhesion and proper sheet resistance.

\subsection{Characterization}

The contact angle of the SWCNT suspension to the PET film was measured using a drop analyzer (DSA100, Krüss). The transmittance of the films was measured using an ultraviolet-visible

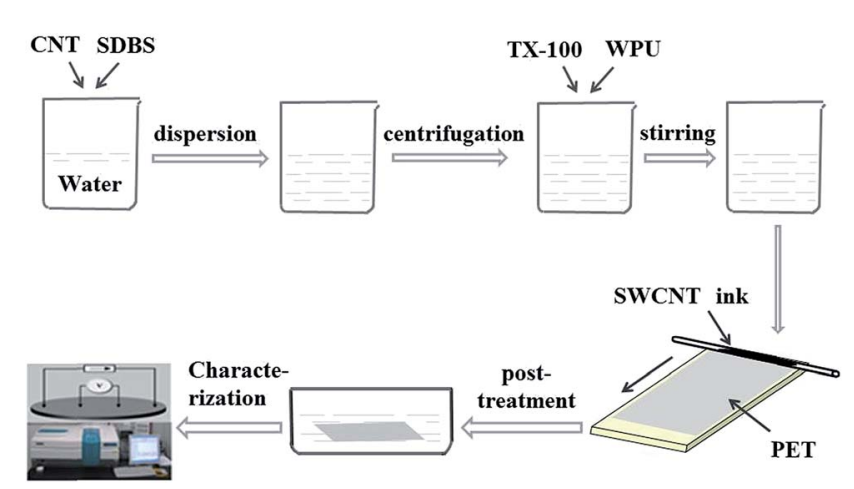

Fig. 1 Schematic about preparation of CNT suspension, coating method, post-treatment and characterization of TAFs. 
(UV-vis) spectrophotometer at the wavelength of $550 \mathrm{~nm}$. The sheet resistance of the film was measured using a four point probe meter (Keithley 2400 SourceMeter, Tektronics). The morphology of the TAFs was observed with afield-emission scanning electron microscope (FE-SEM, S-4800 Hitachi). The EDX spectroscopy was used to determine the residual additives on the surface of the TAFs. A Scotch ${ }^{\mathrm{TM}}$ tape method was used to determine the adhesion of the film.

\section{Results and discussion}

The CNT suspension was spread over the surface of the PET film using a rod coating method with external force, and then the CNT suspension showed contractility because of surface tension. There are some ways to prevent this from happening, for example reducing the surface tension of the suspension or increasing the surface energy of the PET film, but these methods are limited. The optimized dispersion condition was obtained with a mass ratio of SWCNT to SDBS of $1: 10$ as mentioned in a previous report. ${ }^{36}$ The concentration of SWCNT was $1 \mathrm{mg} \mathrm{ml}{ }^{-1}$, so the optimized concentration of SDBS was $10 \mathrm{mg} \mathrm{ml}^{-1}$. The contents of other components were added one after another at the same concentration. The effect of various types of suspension on the contact angle on the PET surface is shown in Fig. 2a. It can be seen that the contact angle decreased greatly after adding SDBS.
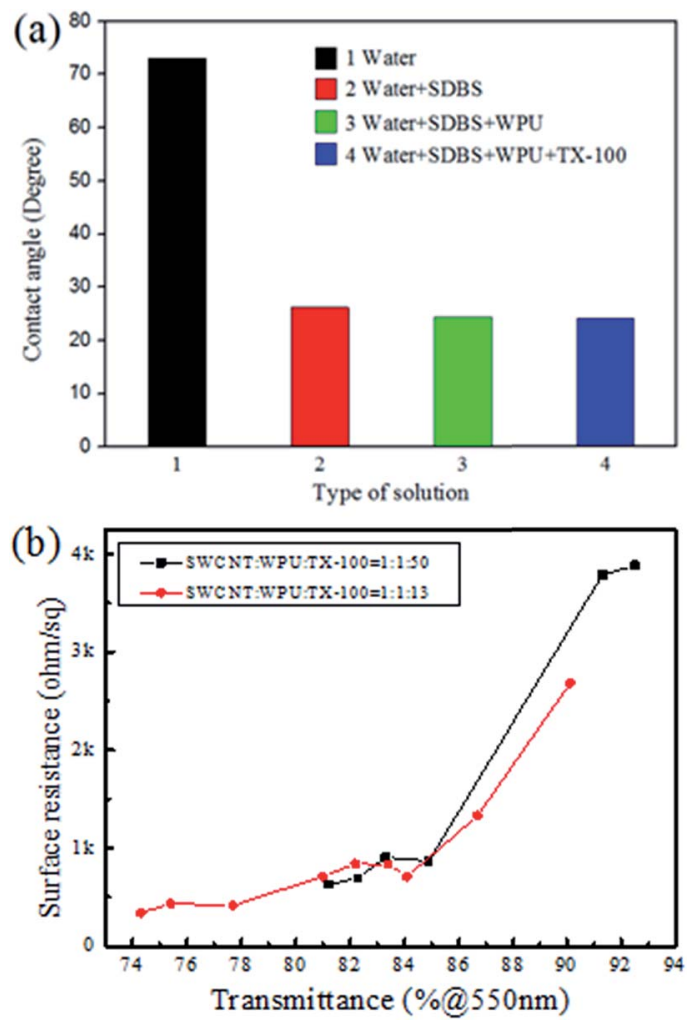

Fig. 2 (a) Effect of different additives on the contact angle of the suspension; (b) the film surface resistance as a function of transmittance with different contents of TX-100, and the points were obtained by changing the dilution of the CNT suspension and the wire diameters.
The contact angle decreased a little after adding WPU, and showed almost no change after further addition of TX-100. The addition of TX-100 increased the viscosity of the CNT suspension and this then increases the dewetting time of the suspension on the PET film substrate, so it then takes more time to dry the liquid film. Fig. $2 b$ shows sheet resistance as a function of transmittance of the film when the TX-100 was added at its minimum amount and this was the usual amount that was used in this research. When the weight ratio of SWCNT, WPU, TX-100 was changed from $1: 1: 13$ to $1: 1: 50$, it can be seen that there is no obvious effect on the sheet resistance and transmittance of the film with different amounts of TX-100.
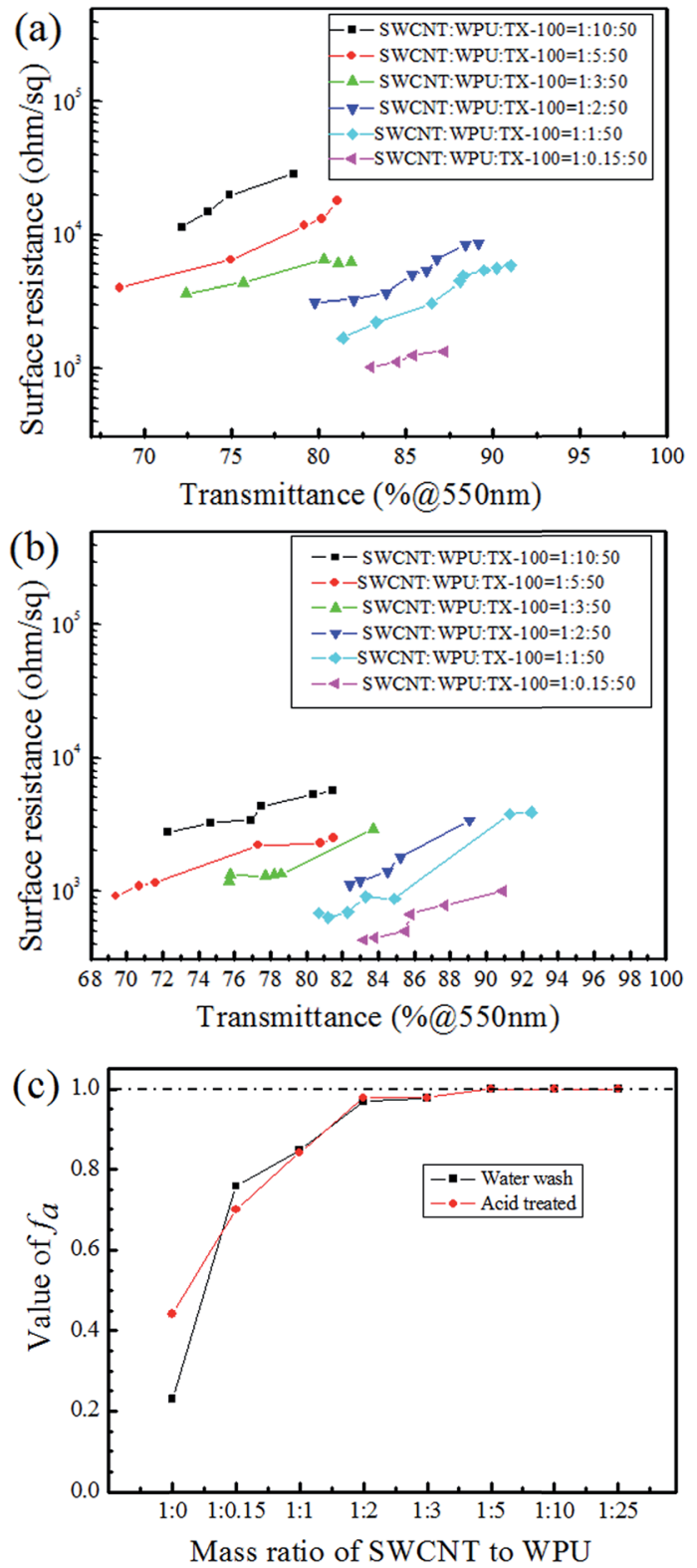

Fig. 3 The relationship between transmittance and sheet resistance of (a) water washed CNT-TAFs and (b) acid treated CNT-TAFs, the points were obtained by changing the dilution of the CNT suspension and wire diameters; (c) adhesion factor $\left(f_{\mathrm{a}}\right)$ at different mass ratios of SWCNT to WPU. 
Depending on the various applications, there are different requirements for transmittance and surface resistance of antistatic films, but the adhesion is very important for all the TAFs in practical applications. In this research, the environmentally friendly WPU adhesive, a linear thermoplastic polymer, was added to improve the adhesion of the TAFs. After rod coating, the CNT-TAFs were rinsed in deionized water and dried at $80^{\circ} \mathrm{C}$ three times, and these were described as water washed CNTTAFs, then these water washed CNT-TAFs were further immersed in nitric acid for $30 \mathrm{~min}$ followed by washing with deionized water to get acid treated CNT-TAFs.

Because of the short time of the acid treatment, the effect of the acid on the PET films was slight. The relationship between the sheet resistance and transmittance of water washed CNTTAFs and acid treated CNT-TAFs at different weight ratios of SWCNT, WPU and TX-100 are shown in Fig. 3a and b, respectively. From Fig. 3a, it can be seen that the content of WPU has

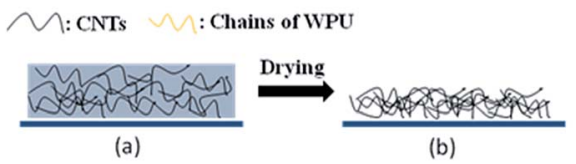

(b)

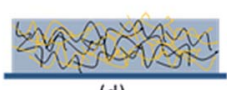

Drying

(d)

(e)

Fig. 4 Schematic for the formation and shape of films made using pure CNT and CNT/WPU. a great influence on the surface resistance of the films. The higher the content of WPU they contain, the greater is the surface resistance. The acid treatment can significantly reduce the surface resistance to less than $10^{3} \Omega$ and this can be seen by comparing Fig. 3b with Fig. 3a. Cao et al. used poly(phenylene sulfide), poly(ether ether ketone) and MWCNTs to fabricate films, but the conductivity of these films was low. ${ }^{37}$ Chou and Cheng utilized MWCNTs and polyimide to make films with a surface resistance of $1.02 \times 10^{6} \Omega \mathrm{cm}^{-2}{ }^{38}$ Obviously, the surface resistance of these films were far higher than the acid treated CNT-TAFs obtained in this research. The adhesion factor $\left(f_{\mathrm{a}}\right)$, which can be easily measured from transmission spectroscopy ${ }^{39}$ was used to characterize the adhesion between SWCNTs and the PET substrate:

$$
f_{\mathrm{a}}=1-\frac{T_{\mathrm{n}}-T_{0}}{100-T_{0}}
$$

where $T_{\mathrm{n}}$ and $T_{0}$ are the transmittance of the detached film after the tape trial and the pristine sample, respectively. Adhesion is good when the value of the $f_{\mathrm{a}}$ is close to 1 . The adhesion factors of these films after water washing and acid treatment are shown in Fig. 3c. It can be seen that if a small amount of WPU is added it can increase the adhesion of the film significantly. The adhesion increased with the increase of WPU content, but the growth trend became less obvious after the weight ratio of SWCNT to WPU was higher than $1: 1$. Meanwhile, the acid treatment has little effect on the adhesion. This shows that the adhesion of CNT-TAFs is good even after acid treatment, indicting a relatively high resistance to acid soaking.

As shown in Fig. 4a and d, the chains of WPU will twist with the CNTs in the mixed solution after stirring and there will exist many contact points between the WPU chains and the CNTs
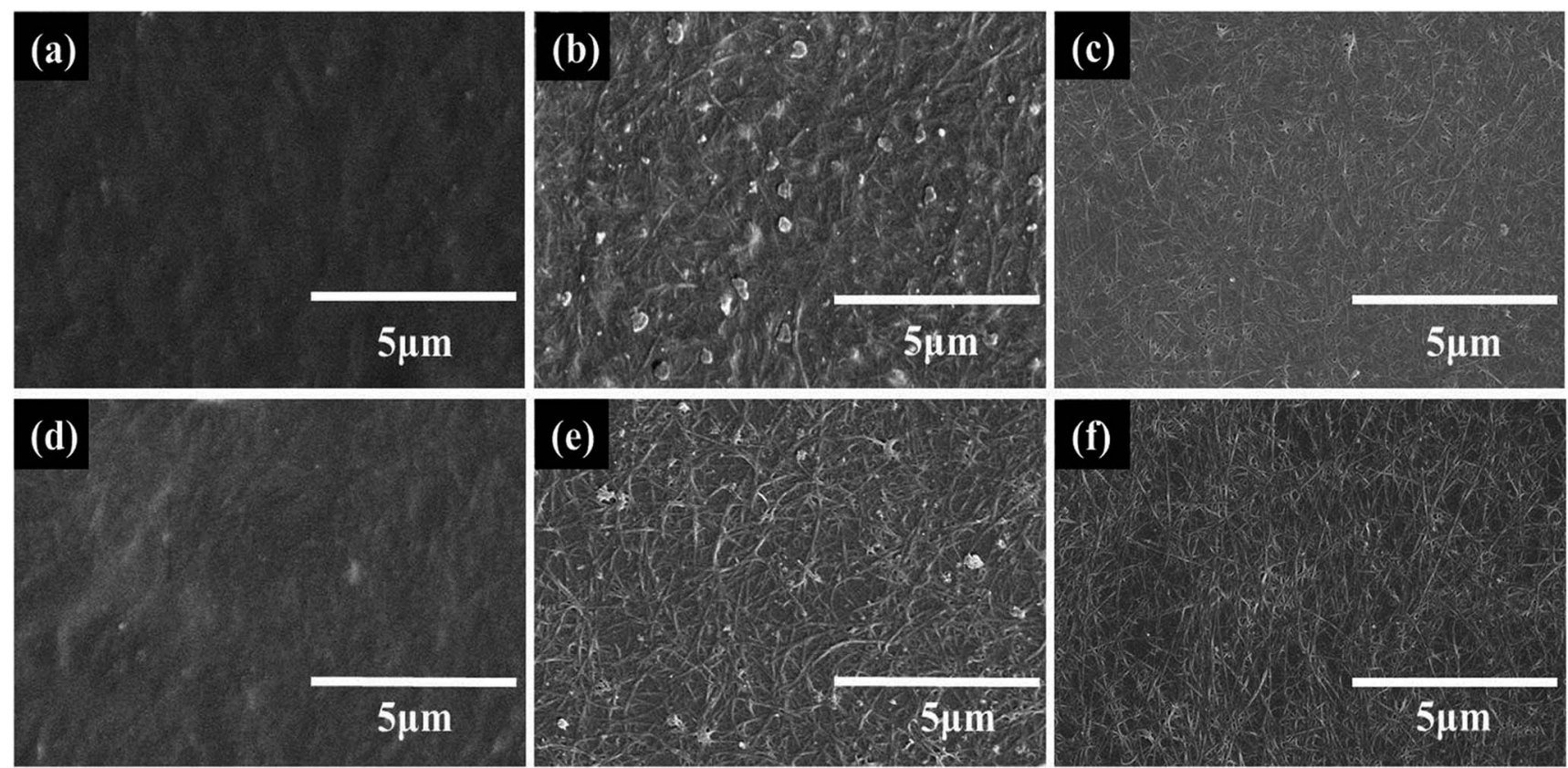

Fig. 5 SEM images of water washed films with SWCNT : WPU (a) $1: 25$, (b) $1: 10$, (c) $1: 1$, and acid treated films with SWCNT : WPU (d) $1: 25$, (e) $1: 10$, (f) $1: 1$ 
that are beneficial for fixing the CNTs. During the process of drying, most of the water will be evaporated and the chains of WPU gradually form a film through interdiffusion ${ }^{\mathbf{4 0}}$ (Fig. $4 \mathrm{e}$ and f). Comparing Fig. 4b, c, e and f, the addition of WPU helps the CNTs to establish more contact points with the PET. Finally, the CNTs are fixed by WPU on the PET substrate. In the tape trial, this mechanism makes the films remain on the surface of PET substrate.

The CNT dispersions were added with different amounts of WPU, which was used as a binder. The more WPU that is added, the higher the adhesion and surface resistance of the CNT film is. After coating, the CNT films on the PET substrates were washed with water and soaked in nitric acid to remove additives and reduce the sheet resistance. Fig. 5 shows the SEM images of water washed and acid treated CNT-TAFs with different contents of WPU and these can be used to determine the surface morphology. By comparing Fig. 5a and b (or Fig. 5d and e) with Fig. 5c (or Fig. 5f), it can be seen that the higher the content of WPU, the more the additives remain on the surface of the TAFs. In particular, when the weight ratio of CNT : WPU is $1: 25$, it is difficult to see the CNTs in Fig. $5 \mathrm{a}$ and d. The surface of the CNT film became cleaner and more tidy after the acid treatment and this can be seen by comparing Fig. $5 \mathrm{c}$ and $\mathrm{f}$ with Fig. $5 \mathrm{~b}$ and e, which explains why the resistance of acid treated film is lower than that of film washed with water as shown in Fig. $3 a$ and $b$. CNTs are responsible for the conductivity and increasing the content of WPU will give a greater surface resistance and higher adhesion. Considering the surface morphology, surface resistance and adhesion of CNTs to PET substrate, the weight ratio of SWCNT to WPU of $1: 1$ was chosen as an optimized solution for CNT-TAFs and the acid treatment was adopted to reduce the sheet resistance. The thicknesses of films, which can be obtained by using atomic force microscopy (AFM) images, along the edge of film are around $50-100 \mathrm{~nm}$ depending on the different transmittances. The films also show a large area uniformity with less $3 \%$ variation in transmittance.

In practice, the antistatic film will inevitably contact with water or be used at high temperature. As is known, for the antistatic film prepared with surfactants, it is easy to lose the antistatic ability in water. To evaluate the water resistance of the optimized TAFs, the films were immersed in water and then taken out to test the sheet resistance after drying every 24 hours.
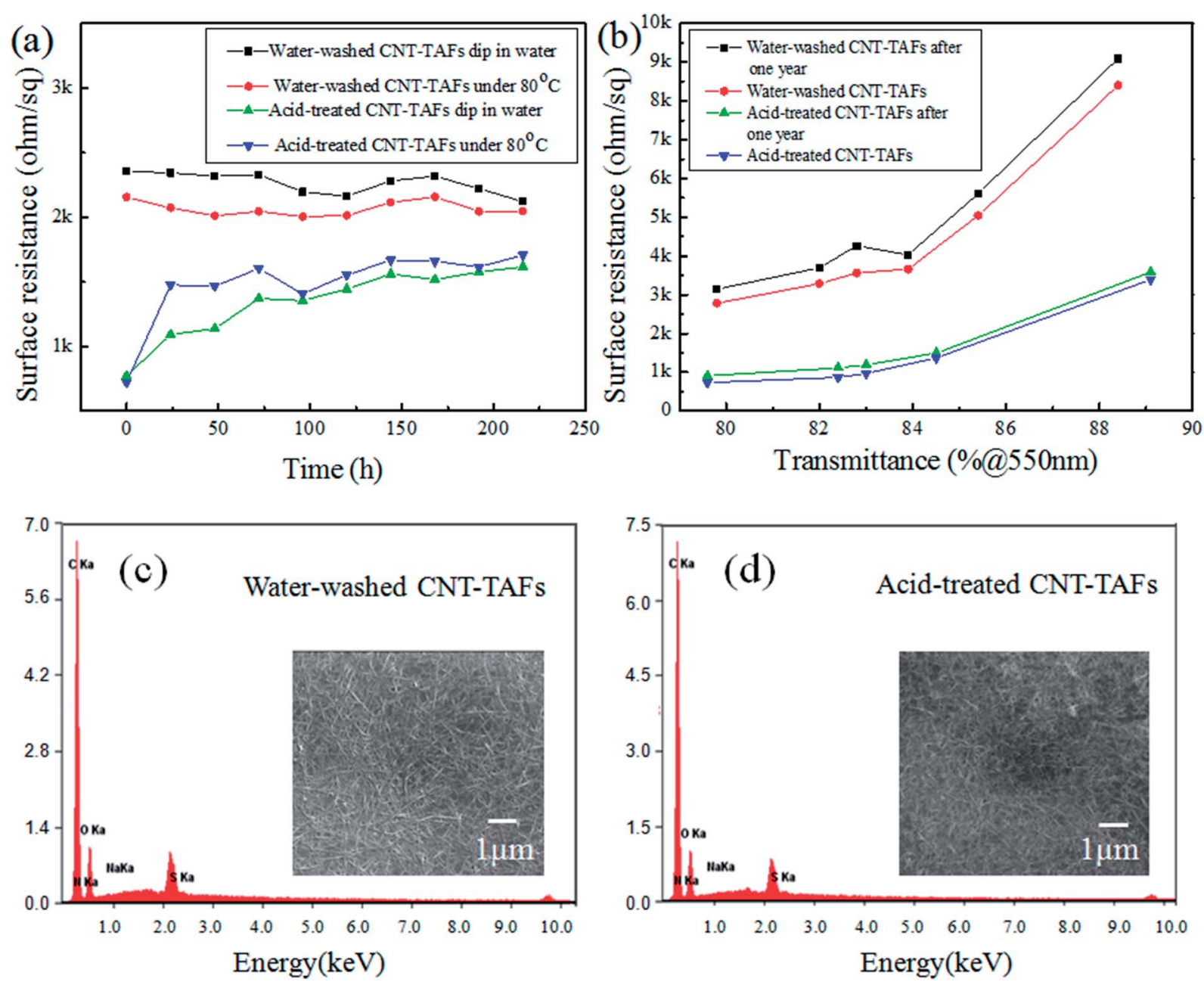

Fig. 6 (a) Influence of the duration of water immersion and high temperature treatment on the sheet resistance; (b) time stability test of water washed CNT-TAFs and acid treated CNT-TAFs, and EDX surface characterization of (c) water washed CNT-TAFs and (d) acid treated CNT-TAFs. 
The results are shown in Fig. 6a. It can be seen that the resistance of water washed CNT-TAFs were still very stable after multiple water immersion tests, which showed good resistance to water. However, the resistance of acid treated CNT-TAFs increased a lot after the first 24 hours and the resistance became stable in the following tests, which also showed good resistance to water. To test the duration of exposure to high temperature, the CNT-TAFs that had been post-treated were placed in an oven with a constant temperature of $80{ }^{\circ} \mathrm{C}$, and then the films were taken out to test their sheet resistance every 12 h. From Fig. 6a, it can be seen that the water washed film has a good duration of high temperature for a long time. The resistance of acid treated CNT-TAFs increased a lot in the first 24 hours, which may because of the dedoping effect. ${ }^{41}$ But the resistance of acid treated CNT-TAFs was very stable after $24 \mathrm{~h}$, and they also showed good resistance to high temperatures. To test the long-term stability of TAFs, a series of water washed CNT-TAFs and acid treated CNT-TAFs were fabricated using the same stable CNT suspension. The content of each component adopted the optimum weight ratio which was $1: 1: 50$ of CNT : WPU : TX-100. Different surface resistances and transmittances were obtained by changing the film thickness. At first, the surface resistance and transmittance of these TAFs were tested immediately, and then the TAFs were put in normal atmospheric environment for more than one year. Finally, the transmittance and surface resistance were tested and compared in the same TAFs group. The resistance and transmittance of the water washed CNT-TAFs and acid treated CNT-TAFs both showed little change after one year as shown in Fig. 6b, which suggests that the films had long-term stability at room temperature. Also, the resistance of acid treated CNT-TAFs were lower than those of water washed CNT-TAFs after a long immersion or high temperature as shown in Fig. 6a, which may be because more dispersants were washed off in the acid treatment as shown in Fig. 6c and d. EDX was conducted to determine the weight ratio changes of the elements using different treatment processes. The element contents are shown in Table 1. In the water washed CNT-TAFs, the films contain CNTs, WPU, TX-100, and SDBS. In the acid treated CNT-TAFs, the films contain CNTs, WPU, TX-100, SDBS, and small amounts of nitric acid. CNTs with a high purity have excellent electrical conductivity after the purification process. ${ }^{42,43}$ There may still be a small amount residual catalyst inside the CNTs after purification, which may cause defects to the CNTs and thus, influence the conductivity of the CNT films. But this will have very little effect on the antistatic application, and it is the CNT content that mainly determines the antistatic property in

Table 1 Element content (wt\%) of TAFs by different treatment process

Element content (wt\%)

\begin{tabular}{llllll} 
Processing method & $\mathrm{C}$ & $\mathrm{O}$ & $\mathrm{S}$ & $\mathrm{N}$ & $\mathrm{Na}$ \\
\hline CNT-TAFs & 69.10 & 23.48 & 2.36 & 2.16 & 2.50 \\
Water washed CNT-TAFs & 72.33 & 24.35 & 0.57 & 2.57 & 0.19 \\
Acid treated CNT-TAFs & 72.97 & 23.66 & 0.37 & 2.91 & 0.10
\end{tabular}

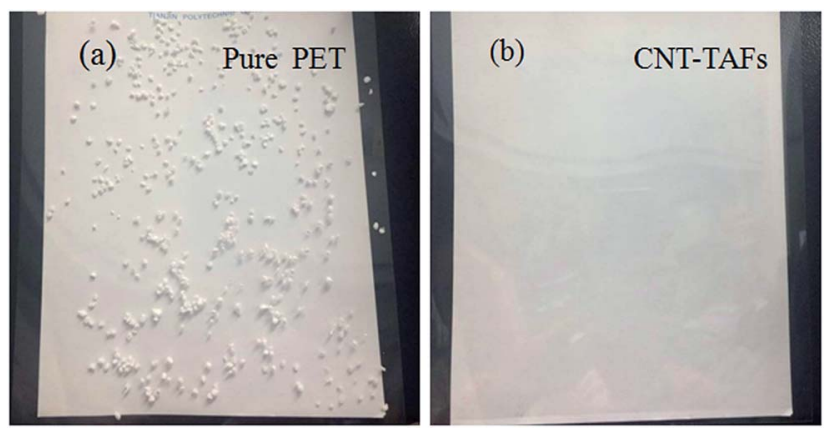

Fig. 7 Antistatic tests of (a) pure PET and (b) acid treated CNT-TAFs.

antistatic films. The sulfur (S) element comes from the SDBS and the amount of the $\mathrm{S}$ element was greatly reduced after washing in water and acid. The nitrogen (N) element comes from nitrate oxidization and WPU, and the amount of the $\mathrm{N}$ element was increased after acid treatment and this may because of nitric acid doping. These results indicate that water and acid treatment can both remove SDBS, and that the acid treatment is more efficient than water treatment to remove the additives on the surface of the CNT films, ${ }^{44}$ so the surface resistance of acid treated CNT-TAFs are lower than those of water washed CNT-TAFs after a long water immersion or high temperature.

The antistatic character was compared between the pure PET film and the acid treated CNT-TAFs as shown in Fig. 7. The two films were abraded and polystyrene microspheres were placed on the two films, then the two films were pulled gently so that they were vertical. The pure PET film adsorbed a lot of polypropylene microspheres as shown in Fig. 7a. However, the acid treated CNT-TAFs did not adsorb any polypropylene microspheres as shown in Fig. 7b. This phenomenon shows that the acid treated CNT-TAFs can be used as an antistatic film with high transmittance.

\section{Conclusion}

High performance SWCNT/WPU/TX-100 hybrid TAFs were fabricated using low power bar coating technology involving different hybrid weight ratios of SWCNT, WPU and TX-100. The addition of WPU can contribute to improve the adhesion of CNTs to the PET film and the content of WPU influence promotion degree. Antistatic films with a high quality need to have an elevated performance in all aspects. Through comprehensive determinations of surface morphology, surface resistance and adhesion of CNTs to PET substrate, a weight ratio of SWCNT to WPU of $1: 1$ was chosen as the optimized solution for TAFs. Washing with water and further acid treatment could effectively remove most of the additives, thereby decreasing the sheet resistance of the films. In addition, these films showed excellent antistatic properties and high stability to water and high temperature. Finally, films were obtained that had a large area, were uniform, were transparent, had low sheet resistance and had strong adhesion at room temperature, and so can be used in wide fields of antistatic applications. 


\section{Conflicts of interest}

There are no conflicts to declare.

\section{Acknowledgements}

The authors gratefully acknowledge financial support from the Natural Science Foundation of Tianjin China (15JCZDJC37900), and the Science and Technology Plans of Tianjin China (16PTSYJC00090).

\section{References}

1 J. Petermann and U. Rieck, J. Polym. Sci., Part B: Polym. Phys., 1987, 25, 279-293.

2 C. Armeniades, I. Kuriyama, J. Roe and E. Baer, J. Macromol. Sci., Part B: Phys., 1967, 1, 777-791.

3 O. Motta, L. Di Maio, L. Incarnato and D. Acierno, Polymer, 1996, 37, 2373-2377.

4 B. Henry, F. Dinelli, K.-Y. Zhao, C. Grovenor, O. Kolosov, G. A. D. Briggs, A. Roberts, R. Kumara and R. Howson, Thin Solid Films, 1999, 355, 500-505.

5 G. A. J. Orchard, P. Spiby and I. M. Ward, J. Polym. Sci., Part B: Polym. Phys., 1990, 28, 603-621.

6 G. S. Ryu, S. H. Jeong, B. C. Park, B. Park and C. K. Song, Org. Electron., 2014, 15, 1672-1677.

7 B. Zimmermann, H. F. Schleiermacher, M. Niggemann and U. Wuerfel, Sol. Energy Mater. Sol. Cells, 2011, 95, 1587-1589.

8 N. Maki, S. Nakano and H. Sasaki, Packag. Technol. Sci., 2004, 17, 249-256.

9 M. Narkis, G. Lidor, A. Vaxman and L. Zuri, J. Electrost., 1999, 47, 201-214.

10 C. Li, T. Liang, W. Lu, C. Tang, X. Hu, M. Cao and J. Liang, Compos. Sci. Technol., 2004, 64, 2089-2096.

11 S. Zhang, C. Wang, H. Yuan, B. Zhang, X. Lin and Z. Lin, J. Electrost., 2013, 71, 1036-1040.

12 A. M. Raspolli Galletti, C. Antonetti, M. Marracci, F. Piccinelli and B. Tellini, Appl. Surf. Sci., 2013, 280, 610618.

13 T. Ohishi, S. Maekawa, T. Ishikawa and D. Kamoto, J. Sol-Gel Sci. Technol., 1997, 8, 511-515.

14 K. Hausmann, Plastics, Addit. Compd., 2007, 9, 40-42.

15 X. Zhou, W. Gao and H. Yang, J. Mater. Sci., 2006, 41, 37153722.

16 T. Iwata, A. Tsurumaki, S. Tajima and H. Ohno, Macromol. Mater. Eng., 2014, 299, 794-798.

17 X. Xiang, X. Huining, G. Yong, L. Shuzhao, W. Dafu and A. Zheng, Macromol. Mater. Eng., 2012, 126, 83-90.

18 S. Konagaya, K. Abe and H. Ishihara, Plast., Rubber Compos., 2002, 31, 201-204.

19 B. Belaabed, S. Lamouri, N. Naar, P. Bourson and S. O. S. Hamady, Plast., Rubber Compos., 2010, 42, 546-554.

20 C.-H. Chen, Y.-T. Kan, C.-F. Mao, W.-T. Liao and C.-D. Hsieh, Surf. Coat. Technol., 2013, 231, 71-76.

21 A. Anson-Casaos, R. Mis-Fernandez, C. M. Lopez-Alled, E. Almendro-Lopez, J. Hernandez-Ferrer, J. M. Gonzalez-
Dominguez and M. T. Martinez, Chem. Eng. Sci., 2015, 138, 566-574.

22 H.-Z. Geng, K. K. Kim, K. Lee, G. Y. Kim, H. K. Choi, D. S. Lee, K. H. An, Y. H. Lee, Y. Chang, Y. S. Lee, B. Kim and Y. J. Lee, Nano, 2007, 2, 157-167.

23 Y. Meng, X.-B. Xu, H. Li, Y. Wang, E.-X. Ding, Z.-C. Zhang and H.-Z. Geng, Carbon, 2014, 70, 103-110.

24 Y. Wang, H.-J. Yang, H.-Z. Geng, Z.-C. Zhang, E.-X. Jing, Y. Meng, Z.-J. Luo, J. Wang, X.-M. Su and S.-X. Da, J. Mater. Chem. C, 2015, 3, 3796-3802.

25 J. A. Robinson, E. S. Snow, S. C. Badescu, T. L. Reinecke and F. K. Perkins, Nano Lett., 2006, 6, 1747-1751.

26 T. A. S. Hameed, A. Aneesh, M. R. Baiju and P. Predeep, Trans. Indian Inst. Met., 2011, 64, 283-285.

27 J. Gao, X. Mu, X. Y. Li, W. Y. Wang, Y. Meng, X. B. Xu, L. T. Chen, L. J. Cui, X. M. Wu and H. Z. Geng, Nanotechnology, 2013, 24, 435201-435209.

28 H.-D. Huang, C.-Y. Liu, L.-Q. Zhang, G.-J. Zhong and Z.-M. Li, ACS Sustainable Chem. Eng., 2015, 3, 317-324.

29 S. W. Yoon, S. Lee, I. S. Choi, Y. Do and S. Park, Macromol. Res., 2015, 23, 713-718.

30 J. H. Kim, J. M. Jung, T. Sultana, J. Y. Kwak, T. K. Hwang and K. T. Lim, Mol. Cryst. Liq. Cryst., 2010, 532, 499-506.

31 Y. Kitamura, H. Tone, M. Nakano and J. Suehiro, Adv. Mater. Res., 2013, 699, 513-518.

32 L. Lei, Z. Xia, G. Cao and L. Zhong, Colloid Polym. Sci., 2013, 292, 527-532.

33 J.-M. Yeh, C.-T. Yao, C.-F. Hsieh, L.-H. Lin, P.-L. Chen, J.-C. Wu, H.-C. Yang and C.-P. Wu, Eur. Polym. J., 2008, 44, 3046-3056.

34 M. Melchiors, M. Sonntag, C. Kobusch and E. Jurgens, Prog. Org. Coat., 2000, 40, 99-109.

35 L. Lei, L. Zhong, X. Lin, Y. Li and Z. Xia, Chem. Eng. J., 2014, 253, 518-525.

36 J. Gao, W.-Y. Wang, L.-T. Chen, L.-J. Cui, X.-Y. Hu and H.-Z. Geng, Appl. Surf. Sci., 2013, 277, 128-133.

37 L. Cao, S. L. Deng and Z. D. Lin, Polymers, 2016, 8, 50-61.

38 S.-C. Chou and Y.-Y. Cheng, Polym. Polym. Compos., 2012, 20, 353-358.

39 S. W. Lee, K. K. Kim, Y. A. N. Cui, S. C. Lim, Y. W. Cho, S. M. Kim and Y. H. Lee, Nano, 2010, 05, 133-138.

40 Z.-F. Wu, H. Wang, M. Xue, X.-Y. Tian, H.-F. Zhou, X.-Z. Ye, K. Zheng and Z.-Y. Cui, Compos. Sci. Technol., 2015, 114, 50-56.

41 H.-Z. Geng, K. K. Kim, C. Song, N. T. Xuyen, S. M. Kim, K. A. Park, D. S. Lee, K. H. An, Y. S. Lee, Y. Chang, Y. J. Lee, J. Y. Choi, A. Benayad and Y. H. Lee, J. Mater. Chem., 2008, 18, 1261-1266.

42 G.-H. Xu, Q. Zhang, J.-Q. Huang, M.-Q. Zhao, W.-P. Zhou and F. Wei, Langmuir, 2010, 26, 2798-2804.

43 S.-X. Da, J. Wang, H.-Z. Geng, S.-L. Jia, C.-X. Xu, L.-G. Li, P.-P. Shi and G. F. Li, Appl. Surf. Sci., 2017, 392, 1117-1125. 44 H.-Z. Geng, K. K. Kim, K. P. So, Y. S. Lee, Y. Chang and Y. H. Lee, J. Am. Chem. Soc., 2007, 129, 7758-7759. 